\title{
Non smooth evolution models in crowd dynamics: mathematical and numerical issues
}

\author{
Bertrand Maury* \\ * Laboratoire de Mathématique d'Orsay, Université Paris-Sud, 91405 Orsay, \\ France
}

\begin{abstract}
Accounting for hard congestion in crowd motion modeling leads to non-smooth evolution problems. At the microscopic level (individuals are represented separately), these problems fit in the framework of non smooth analysis in Hilbert spaces, and the tools developed in the 70's to handle the so-called sweeping process are directly adaptable. At the macroscopic scale (the population is represented by a density), a similar approach can be carried out. This is done by identifying densities with measures in the Wasserstein space, endowed with the distance based on optimal transportation for the quadratic cost. These lecture notes provide an introduction to the mathematical theory of these models, and a description of numerical methods, in the microscopic (ODE) and macroscopic (PDE) cases.
\end{abstract}

\section{Introduction}

Congestion is a crucial issue in crowd motion modeling. When people want to evacuate a room they all head to the door, which tends to decrease their mutual distance, thereby increasing the local density. Investigating a bit further these considerations will shed light on the different aspects of congestion, in the microscopic and in the macroscopic settings. As a first step, consider people in a corridor, and assume that they all want to go to the right direction. The situation is likely to become critical in terms of density if the velocities decrease from left to right (i.e. the people in front move slower than the people behind them), whereas increasing velocities will tend to relax the situation (the density decreases). In the two-dimensional setting, the notion of "increasing" velocity field has to be extended in some way. Consider a velocity field $\mathbf{U}$ in the plane, and two individuals 1 and 2 , identified to rigid discs centered at $\mathbf{q}_{1}$ and $\mathbf{q}_{2}$. Each individual $\mathbf{q}_{i}$ is assumed to move at velocity $\mathbf{U}\left(\mathbf{q}_{i}\right)$. Their distance increases whenever

$$
\left(\mathbf{U}\left(\mathbf{q}_{2}\right)-\mathbf{U}\left(\mathbf{q}_{1}\right)\right) \cdot\left(\mathbf{q}_{2}-\mathbf{q}_{1}\right) \geq 0 .
$$


A velocity field that verifies (1) is said to be monotone (which actually means increasing in a general, multidimensional sense). An archetypal monotone field is $\mathbf{x} /|\mathbf{x}|$. The velocity field corresponding to the evacuation of a room (the desired velocity is directed to a single "point", which is the door) is roughly the opposite of this field: the evolution according to this field tends to decrease all distances. Let us go a bit further in the classification between relaxing and non relaxing fields. The monotony (1) condition above can be written for $\mathbf{q}_{2}$ seen as a variation of $\mathbf{q}_{1}$, i.e. $\mathbf{q}_{2}=\mathbf{q}_{1}+\varepsilon \mathbf{h}$. Having $\varepsilon$ go to 0 , we obtain that

$$
\mathbf{h} \cdot \nabla \mathbf{U} \cdot \mathbf{h} \geq 0
$$

for any direction $\mathbf{h}$. Note that it can be expressed in terms of the symmetrized gradient of $\mathbf{U}$, i.e. $\nabla \mathbf{U}+{ }^{t} \nabla \mathbf{U}$, since the skew symmetric part does not contribute. Now consider particles (or individuals) evolving according to this velocity fields. The velocity of a point $\mathbf{q}+\varepsilon \mathbf{h}$ close to a reference point $\mathbf{q}$ writes

$$
\mathbf{U}(\mathbf{q}+\varepsilon \mathbf{h})=\mathbf{U}(\mathbf{q})+\varepsilon\left(\frac{\nabla \mathbf{U}-{ }^{t} \nabla \mathbf{U}}{2}\right) \cdot \mathbf{h}+\varepsilon\left(\frac{\nabla \mathbf{U}+{ }^{t} \nabla \mathbf{U}}{2}\right) \cdot \mathbf{h}+o(\varepsilon),
$$

which means that the local velocity around $\mathbf{q}$ is mainly a rigid motion composed of a translation (velocity $\mathbf{U}(\mathbf{q})$ ), a rotation (angular velocity $\partial_{1} u_{2}-\partial_{2} u_{1}$ ), and a last component which corresponds to deformations. The corresponding tensor (or matrix)

$$
\mathbf{e}=\frac{\nabla \mathbf{U}+{ }^{t} \nabla \mathbf{U}}{2}
$$

is called the strain tensor. It is symmetric, thereby diagonalizable, with real eigenvalues, the sign of which indicate the nature of the deformation: a negative value correspond to compression in the corresponding direction, whereas a positive one reflects expansion (i.e. distances increase). Condition (1) therefore expresses that all eigenvalues of $\mathbf{e}$ are nonnegative, which means that the flow is expanding in all directions. Coming back to the problem of congestion, it means that problems can be expected (i.e. some distances between individuals may decrease down to physical contact) as soon as the desired velocity field is not expanding in all directions. Actually, the "problems" we mentioned concern safety (i.e. jamming can be expected upstream the exit, which may tend to increase the evacuation time and induce casualties), but the fact that the field concentrates people makes the problem easier from the mathematical standpoint, as far as the uncontested situation is concerned. Indeed, if $\mathbf{-} \mathbf{U}$ is monotone, as it is expected 
for an evacuation through a small exit, then the evacuation process (without congestion) can be written

$$
\frac{d \mathbf{q}}{d t}-\mathbf{U} \ni 0
$$

and well-posedness (existence and uniqueness of a solution) can be established without assuming that $\mathbf{U}$ is Lipschitz, as the standard theory of Ordinary Differential Equations requires (see e.g. Bauschke and Combettes (2011)).

Let us now point out a deep difference between the microscopic approach that we followed previously and the macroscopic one. The latter consists in representing the population by a density $\rho(x, t)$. Still denoting by $\mathbf{U}$ the underlying velocity field ${ }^{1}$, the "people conservation" in any domain $\omega$ expresses the time derivative of the population in $\omega$ and the flux through its boundary, and it writes

$$
\frac{d}{d t} \int_{\omega} \rho=\int_{\omega} \frac{\partial \rho}{\partial t}=-\int_{\partial \omega} \rho \mathbf{U} \cdot \mathbf{n}=-\int_{\omega} \nabla \cdot(\rho \mathbf{U}) .
$$

Since conservation holds for any such subdomain, we have the transport equation

$$
\frac{\partial \rho}{\partial t}+\nabla \cdot(\rho \mathbf{U})=0
$$

The derivative of $\rho$ along a trajectory (total derivative) writes

$$
\frac{\partial \rho}{\partial t}+\mathbf{U} \cdot \nabla \rho=-\rho \nabla \cdot \mathbf{U}
$$

which is nonpositive (i.e. the density relaxes toward a smaller value) as soon as $\nabla \cdot \mathbf{U} \geq 0$. Note that the condition is weaker than the microscopic one: preserving nondecreasing distance necessitates expansion in all directions (the eigenvalues of $\mathbf{e}$ are nonnegative), whereas here only the sign of the trace (that is the divergence of $\mathbf{U}$, and also the sum of eigenvalues of the strain tensor e defined by (2)) is relevant. As we shall see in Section 2, handling the constraints at the microscopic level consists in accounting for the concentrating character of the velocity field, neither in all directions (as in the first approach we presented), nor in a mean sense like in the macroscopic setting, but rather in some particular directions that correspond to the actual contacts between individuals. Those directions depend

\footnotetext{
${ }^{1}$ We shall make later on a difference between the desired velocity field and the actual velocity field; the latter accounts for constraints.
} 
on the local structure of the contact network, and the native heterogeneity of jammed population will induce numerical difficulties. The macroscopic problem is easier from this standpoint, since the problem is homogeneous: the constraint to remain below a prescribed value is the same over the whole saturated zone. The difficulty here is rather due to the Eulerian character of Partial Differential Equations, which rules out the possibility to directly apply tools of convex analysis, at least in standard functional spaces. We shall details in Section 3 how the Wasserstein Distance on measures will make it possible to adapt some tools that have been developped in Hilbert spaces.

\section{Hard congestion in the microscopic setting}

\subsection{The model}

We consider $N$ individuals identified to rigid disks of radius $r$ in a room identified to a domain $\Omega$. The positions are represented by a vector $\mathbf{q}$ of $\mathbb{R}^{2 N}$ :

$$
\mathbf{q}=\left(\mathbf{q}_{1}, \mathbf{q}_{2}, \cdots, \mathbf{q}_{N}\right) \in \mathbb{R}^{2 N}
$$

The distance between two individuals $i$ and $j$ is denoted by

$$
D_{i j}=\left|\mathbf{q}_{j}-\mathbf{q}_{i}\right|-2 r .
$$

The set of feasible configurations (contact is allowed, but no overlapping) can be written

$$
K=\left\{\mathbf{q} \in \mathbb{R}^{2 N}, D_{i j} \geq 0 \quad \forall i \neq j\right\} .
$$

The distance $D_{i j}$ can be considered as a function of the configuration vector $\mathbf{q}$ (although it depends on $i$ and $j$ only), and we denote by $\mathbf{G}_{i j} \in \mathbb{R}^{2 N}$ its gradient. Ruling out overlapping between two individual amounts to require that their instantaneous relative velocity is nonnegative in the normal direction, as soon as there is contact. It leads to the following definition of the set of feasible (or admissible) velocities ${ }^{2}$

$$
C_{K}(\mathbf{q})=\left\{\mathbf{v} \in \mathbb{R}^{2 N}, D_{i j}(\mathbf{q})=0 \Longrightarrow \mathbf{G}_{i j} \cdot \mathbf{v} \geq 0\right\} .
$$

Now consider that a collection of desired velocities

$$
\mathbf{U}=\left(\mathbf{U}_{1}, \ldots, \mathbf{U}_{N}\right)
$$

\footnotetext{
${ }^{2}$ In order to alleviate notations, we disregard here the constraints that are due to obstacles (walls or piles), but they can be integrated in the set of feasible velocities like inter individual non overlapping constraints.
} 
is given. Following Maury and Venel (2011), we assume that, at each instant, the actual velocity field $\mathbf{u}$ is the closest (in the $\ell^{2}$ sense) to the desired velocity field $\mathbf{U}$, among all feasible fields, i.e.

$$
\mathbf{u}=\frac{d \mathbf{q}}{d t}=P_{C_{K}(\mathbf{q})}(\mathbf{U}) .
$$

The desired velocity $\mathbf{U}_{i}$ may depend on time, on the position of other individuals (if one aims at accounting for social effects). If one considers that the desired velocity of an individual depends on its location only, and that individual are interchangeable, $\mathbf{U}_{i}$ can be defined as $\mathbf{U}_{0}\left(\mathbf{q}_{i}\right)$, where $\mathbf{U}_{0}$ is a desired velocity field, the same for everybody. We consider here the general case: the individual desired velocity depends on all the positions, and this dependence may vary from an individual to the other. Thus, the desired velocity is written $\mathbf{U}=\mathbf{U}(\mathbf{q})$.

\subsection{General setting, catching up approach}

The basic tool to reformulate Model (4) is the decomposition of a Hilbert space according to mutually polar cones, as proposed by Moreau (1962). Consider a closed convex cone $C$ pointed at the origin, in a Hilbert space $H$, i.e.

$$
C \subset H, \mathbb{R}^{+} C \subset C, \lambda x+(1-\lambda) y \in C \quad \forall x, y \in C, \lambda \in[0,1], \bar{C}=C .
$$

The polar cone to $C$ is defined as

$$
C^{\circ}=\{y \in H,(y, x) \leq 0 \quad \forall x \in C\} .
$$

The decomposition of any Hilbert space as the direct sum of a closed vector set and its orthogonal can be extended to cones (Moreau (1962)):

$$
I_{d}=P_{C}+P_{C^{\circ}},
$$

where $I_{d}$ is the identity, and $P_{C}\left(\right.$ resp. $\left.P_{C^{\circ}}\right)$ is the projection on $C$ (resp. $\left.C^{\circ}\right)$.

Eq. (4) can be rewritten

$$
\mathbf{u}=\frac{d \mathbf{q}}{d t}=\mathbf{U}-P_{C_{K}^{\circ}(\mathbf{q})}(\mathbf{U}),
$$

which implies $\mathbf{u}-\mathbf{U} \in-C_{K}^{\circ}(\mathbf{q})$. The latter inclusion is usually written

$$
\frac{d \mathbf{q}}{d t}+N_{K}(\mathbf{q}) \ni \mathbf{U}(\mathbf{q})
$$

where $N_{K}=C_{K}^{\circ}(\mathbf{q})$ is the so-called outward normal cone to $\mathcal{K}$ at $\mathbf{q}$. As we shall see, this inclusion actually characterizes the evolution (i.e. no information is lost by replacing the projection by a simple inclusion). 
Catching up algorithm in the convex case Now assume for a moment that the set $K$ of feasible configurations is convex ${ }^{3}$. By characterization of the projection on a closed convex set, i.e.

$$
\mathbf{q}=P_{K} \tilde{\mathbf{q}} \Longleftrightarrow(\tilde{\mathbf{q}}-\mathbf{q}, \mathbf{z}-\mathbf{q}) \leq 0 \quad \forall \mathbf{z} \in K,
$$

we obtain that the outward normal cone $N_{K}(\mathbf{q})=C_{K}^{\circ}(\mathbf{q})$ can be expressed

$$
N_{K}(\mathbf{q})=\left\{\tilde{\mathbf{q}}-\mathbf{q}, \mathbf{q}=P_{K} \tilde{\mathbf{q}}\right\} .
$$

This suggests the following time discretization, which can be seen as a semi-implicit Euler scheme applied to (5). This strategy was introduced by Moreau (1977) as a catching-up approach to build solutions to similar problems (sweeping process).

Considering a time step $\tau>0$, it consists in discretizing (5) as

$$
\frac{\mathbf{q}^{n+1}-\mathbf{q}^{n}}{\tau}-\mathbf{U}\left(\mathbf{q}^{n}\right) \in-N_{K}\left(\mathbf{q}^{n+1}\right),
$$

which can be written

$$
\mathbf{q}^{n}+\tau \mathbf{U}\left(\mathbf{q}^{n}\right)-\mathbf{q}^{n+1} \in N_{K}\left(\mathbf{q}^{n+1}\right)
$$

By (7), it is equivalent to (Catching-up scheme)

$$
\mathbf{q}^{n+1}=P_{K}\left(\mathbf{q}^{n}+\tau \mathbf{U}\left(\mathbf{q}^{n}\right)\right),
$$

so that the semi-implicit Euler scheme turns out to be explicit in this regard: it reduces to a projection of a predicted position $\tilde{\mathbf{q}}^{n+1}=\mathbf{q}^{n}+\tau \mathbf{U}\left(\mathbf{q}^{n}\right)$ on $K$.

Theoretical issues (convex case) The catching-up algorithm can be used to build a solution to our problem, more precisely its formulation (6). We shall state it in an abstract form.

Proposition 2.1. Let $H$ be a Hilbert space, $K \subset H$ a closed convex set, and

$$
\mathbf{U}: \mathbf{q} \in H \longmapsto \mathbf{U}(\mathbf{q})
$$

\footnotetext{
${ }^{3}$ This assumption is very strong, and rarely verified in realistic situations in the context of crowd motions. It corresponds to the case of a single person in a convex room with no door (!), or to the one-dimensional situation: $N$ individual in a corridor, the size of which is exactly the diameter of individuals.
} 
a Lipschitz function in $H$, bounded over $H$. Consider an initial condition $\mathbf{q}^{0}, T>0 \tau=T / M>0$ a time step, and

$$
\mathbf{q}_{\tau}^{0}, \mathbf{q}_{\tau}^{1}, \ldots, \mathbf{q}_{\tau}^{M},
$$

the elements obtained by application of the Catching-Up algorithm (9). Denoting by $\mathbf{q}_{\tau}$ the corresponding continuous, piecewise affine trajectory, $\mathbf{q}_{\tau}$ converges uniformly toward a solution $t \mapsto \mathbf{q}(t)$ to (6), i.e.

$$
\frac{d \mathbf{q}}{d t}+N_{K}(\mathbf{q}) \ni \mathbf{U}(\mathbf{q}(t)) \text {, for almost every } t \in(0, T) .
$$

Proof. We shall simply give here the main arguments of the proof, and we refer to Venel (2011) for further details. The main argument is a characterization of the outward normal cone for a convex set $K$ (see e.g. Venel (2011)):

$$
\begin{aligned}
\mathbf{v} \in N_{K}(\mathbf{q}) & \Longleftrightarrow \forall \boldsymbol{\xi},\langle\mathbf{v}, \boldsymbol{\xi}\rangle \leq|\mathbf{v}| d_{K}(\mathbf{q}+\boldsymbol{\xi}) \\
& \Longleftrightarrow \quad \exists C>0, \eta>0, \forall \boldsymbol{\xi},|\boldsymbol{\xi}| \leq \eta,\langle\mathbf{v}, \boldsymbol{\xi}\rangle \leq C d_{K}(\mathbf{q}+\boldsymbol{\xi}),
\end{aligned}
$$

where $d_{K}(\cdot)$ denotes the distance to $K$.

The rest of the proof relies on compactness arguments. First of all $\left(\mathbf{q}_{\tau}\right)$ is uniformly bounded in $W^{1, \infty}(0, T)$, so that (up to the extraction of a subsequence) $\left(\mathbf{q}_{\tau}\right)_{\tau}$ converges uniformly toward some trajectory $\mathbf{q}$, and the corresponding velocity $\mathbf{u}_{\tau}=d \mathbf{q}_{\tau} / d t$ (which is piecewise constant) converges toward some $\mathbf{u}$ in the $L^{\infty}$ weak-star topology.

It holds that

$$
\mathbf{q}_{\tau}^{n+1}=P_{K}\left(\mathbf{q}_{\tau}^{n}+\tau \mathbf{U}\left(\mathbf{q}_{\tau}^{n}\right)\right),
$$

which implies

$$
\left(\mathbf{q}_{\tau}^{n}+\tau \mathbf{U}\left(\mathbf{q}_{\tau}^{n}\right)\right)-\mathbf{q}_{\tau}^{n+1} \in N_{K}\left(\mathbf{q}_{\tau}^{n+1}\right),
$$

i.e.

$$
\frac{d \mathbf{q}_{\tau}}{d t}-\mathbf{U}\left(\tilde{\mathbf{q}}_{\tau}\right) \in-N_{K}\left(\overline{\mathbf{q}}_{\tau}\right) \text {, for a.e. } t \in(0, T),
$$

where $\tilde{\mathbf{q}}_{\tau}$ and $\overline{\mathbf{q}}_{\tau}$ are piecewise constant approximate solutions, with value at the beginning and at the end of time intervals, respectively. Notice that both sequences $\tilde{\mathbf{q}}_{\tau}$ and $\overline{\mathbf{q}}_{\tau}$ uniformly converge to $\mathbf{q}$. By (10), the previous inclusion implies

$$
-\left\langle\frac{d \mathbf{q}_{\tau}}{d t}-\mathbf{U}\left(\tilde{\mathbf{q}}_{\tau}\right), \boldsymbol{\xi}\right\rangle \leq \underbrace{\left|\frac{d \mathbf{q}_{\tau}}{d t}-\mathbf{U}\left(\tilde{\mathbf{q}}_{\tau}\right)\right|}_{\leq M} d_{K}(\overline{\mathbf{q}}+\boldsymbol{\xi}) .
$$


Since $\mathbf{u}_{\tau}-\mathbf{U}\left(\tilde{\mathbf{q}}_{\tau}\right)$ converges weakly-star to $\mathbf{u}-\mathbf{U}\left(\mathbf{q}_{\tau}\right)$ (in $L^{\infty}$ ), by Mazur's Lemma, a convex combination $\mathbf{z}_{\tau}$ of the sequence $\left(\mathbf{u}_{\tau}-\mathbf{U}\left(\tilde{\mathbf{q}}_{\tau}\right)\right)$ converges strongly in $L^{1}$ toward $\mathbf{u}-\mathbf{U}(\mathbf{q})$. We therefore have point wise convergence of $\mathbf{z}_{\tau}$ toward $\mathbf{u}-\mathbf{U}(\mathbf{q})$ for almost every time $t$. Thus, for any time at which pointwise convergence of $\mathbf{z}_{\tau}$ holds,

$$
\lim \sup \left\langle\mathbf{z}_{\tau}, \boldsymbol{\xi}\right\rangle \leq M d_{K}(\mathbf{q}+\boldsymbol{\xi})
$$

so that.

$$
\left\langle\frac{d \mathbf{q}}{d t}-\mathbf{U}(\mathbf{q}), \boldsymbol{\xi}\right\rangle \leq M d_{K}(\mathbf{q}+\boldsymbol{\xi}) .
$$

By using again (10) (second characterization) the other way around, we obtain

$$
\frac{d \mathbf{q}}{d t}-\mathbf{U}(\mathbf{q}) \in-N_{K}(\mathbf{q}) \text { for a.e. } t
$$

which ends the proof.

Crowd motion model: the non convex situation As for the crowd motion model we introduced, the feasible set $K$ is not convex as soon as there are two individuals (i.e. rigid discs). Yet, as suggested by the previous approach, similar results can be expected as soon as it is possible to project the predicted configuration $\tilde{\mathbf{q}}^{n+1}=\mathbf{q}^{n}+\tau \mathbf{U}\left(\mathbf{q}^{n}\right)$ on $K$. More precisely, if $\mathbf{q}^{n}$ is feasible (i.e. in $K$ ), if $\mathbf{U}$ is bounded, then a control on the time step ensures that $\tilde{\mathbf{q}}^{n+1}$ is close to $K$. It is therefore enough to prescribe that the projection is well-defined in the neighborhood of $K$. This leads to the notion of prox-regular sets (Poliquin and Rockafellar (1996)). Let us first extend the notion of outward normal cone to non convex sets (Clarke et al. (1995)):

Definition 2.2. Let $K \subset H$ be a closed set, and $\mathbf{q} \in K$. The outward normal cone to $K$ at $\mathbf{q}$ is defined by

$$
N_{K}(\mathbf{q})=\left\{\mathbf{v} \in H, \exists \alpha>0, \mathbf{q} \in P_{K}(\mathbf{q}+\alpha \mathbf{v})\right\},
$$

where $P_{K}(\tilde{\mathbf{q}})$ denotes here the set of elements of $H$ that realize the distance between $\tilde{\mathbf{q}}$ and $K$.

The prox-regularity is defined as follows:

Definition 2.3. Let $K \subset H$ be a closed set, and $\eta>0$. The set $K$ is said to be uniformly $\eta$ prox-regular if, for any $\mathbf{q} \in \partial K, N_{K}(\mathbf{q})$ is not reduced to $\{0\}$ and, for any $\mathbf{v} \in N_{K}(\mathbf{q})$, with $|\mathbf{v}|=1$, we have

$$
B(\mathbf{q}+\eta \mathbf{v}, \eta) \cap K=\emptyset .
$$


A weakened form of the characterization (10) holds for prox-regular sets:

$$
\begin{gathered}
\mathbf{v} \in N_{K}(\mathbf{q}) \Longleftrightarrow \\
\exists \alpha>0, C>0, \forall \boldsymbol{\xi},|\boldsymbol{\xi}|<\alpha,\langle\mathbf{v}, \boldsymbol{\xi}\rangle \leq C d_{K}(\mathbf{q}+\boldsymbol{\xi})+\frac{|\mathbf{v}|}{2 \eta}|\boldsymbol{\xi}|^{2},
\end{gathered}
$$

Thanks to this characterization, Prop. 2.1 can be extended to the prox regular case, which provides a framework for the crowd motion model, since the feasible set $K$ can be shown to be prox-regular (Maury and Venel (2011)).

Saddle point formulation The outward normal cone $N_{K}(\mathbf{q})=C_{K}^{\circ}(\mathbf{q})$ can be parametrized thanks to Farkas' Lemma (see e.g. Rockafellar (1970), p. 200):

$$
\begin{aligned}
N_{K}(\mathbf{q}) & =\left\{\mathbf{v} \in \mathbb{R}^{2 N}, D_{i j}(\mathbf{q})=0 \Longrightarrow \mathbf{G}_{i j} \cdot \mathbf{v} \geq 0\right\}^{\circ} \\
& =\left\{-\sum_{i<j} \lambda_{i j} \mathbf{G}_{i j}, \lambda_{i j} \geq 0, D_{i j}>0 \Rightarrow \lambda_{i j}=0\right\} .
\end{aligned}
$$

As a consequence, the projection of $\mathbf{U}$ on $N_{K}(\mathbf{q})$ can be formulated in a saddle point manner. It amounts to find $\left(\lambda_{i j}\right)_{(i, j) \in \Lambda}$, where $\Lambda$ is the set of $(i, j)$ such that the constraint is active (i.e. $D_{i j}(\mathbf{q})=0$ ), and $\mathbf{u} \in \mathbb{R}^{2 N}$, such that

$$
\left\{\begin{array}{l}
\mathbf{u}+B^{\star} \lambda=\mathbf{U} \\
B \mathbf{u} \leq 0 \\
\lambda \\
(B \mathbf{u}, \lambda)=0
\end{array}\right.
$$

where $B$ is a matrix, each line of which corresponds to the constraint

$$
-\mathbf{G}_{i j} \cdot \mathbf{u} \leq 0, \quad(i, j) \in \Lambda,
$$

with

$$
\mathbf{G}_{i j}=\left(0, \ldots, 0,-\mathbf{e}_{i j}, 0, \ldots, 0, \mathbf{e}_{i j}, 0, \ldots, 0\right), \mathbf{e}_{i j}=\frac{\mathbf{q}_{j}-\mathbf{q}_{i}}{\left|\mathbf{q}_{j}-\mathbf{q}_{i}\right|} .
$$

Thus, the projection takes the form of a (unilateral) discrete Darcy problem. This analogy is not only formal. Consider for example the case of people in a corridor (one-dimensional setting): 


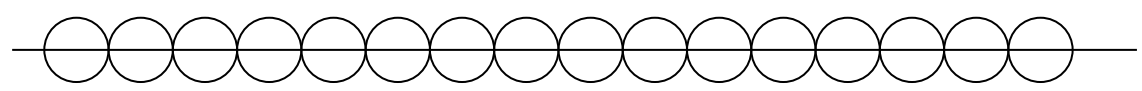

The gradients are

$$
\mathbf{G}_{12}=(-1,1,0, \ldots, 0), \mathbf{G}_{23}=(0,-1,1,0, \ldots, 0), \text { etc. }
$$

so that the constraint matrix writes

$$
B=\left(\begin{array}{ccccc}
1 & -1 & 0 & \ldots & \ldots \\
0 & 1 & -1 & \ldots & \ldots \\
0 & 0 & \ddots & \ddots & \ldots \\
0 & 0 & \ldots & 1 & -1
\end{array}\right)
$$

that is the discrete counterpart of $-\partial_{x}$ (opposite of the divergence), and $B^{\star}$ corresponds to $\partial_{x}$ (gradient). Note that $B B^{\star}$ is the discrete counterpart of the one-dimensional Laplacian $-\partial_{x x}$. This observation reveals the numerical underlying difficulties: at each time step, the computation of the actual velocity can be expected to be, at least, as difficult as solving a discrete Poisson problem with the same number of degrees of freedom. It also illustrates the non-local effect of the projection onto the set of feasible velocities: all individuals gathered in a same cluster are likely to interact.

The projection (9) can also be formulated in a dual manner: since

$$
\mathbf{q}^{n+1}=P_{K}\left(\mathbf{q}^{n}+\tau \mathbf{U}\left(\mathbf{q}^{n}\right)\right)
$$

there exists a collection of Lagrange multipliers $\left(\lambda_{i j}\right)_{(i, j) \in \Lambda}$ such that

$$
\mathbf{q}^{n}+\tau \mathbf{U}\left(\mathbf{q}^{n}\right)=\mathbf{q}^{n+1}-\sum_{i, j} \lambda_{i j} \mathbf{G}_{i j}\left(\mathbf{q}^{n+1}\right),
$$

which can be written

$$
\frac{\mathbf{q}^{n+1}-\mathbf{q}^{n}}{\tau}+B^{\star} \lambda=\mathbf{U}\left(\mathbf{q}^{n}\right) .
$$

Yet, in spite of its formal simplicity, this formulation does not directly lead to a tractable numerical scheme, since the matrix $B$ (together with the set $\Lambda$ of active couples) depends on the unknown $\mathbf{q}^{n+1}$. It reflects the implicit (and highly nonlinear) character of the scheme.

\section{$2.3 \quad$ Numerical scheme}

In order to obtain a tractable numerical scheme, we replace (as in Maury (2006)) the set of feasible configurations by some kind of local inner approximation. More precisely, considering a given configuration $\mathbf{q} \in K$, we introduce (see Fig. 1) 


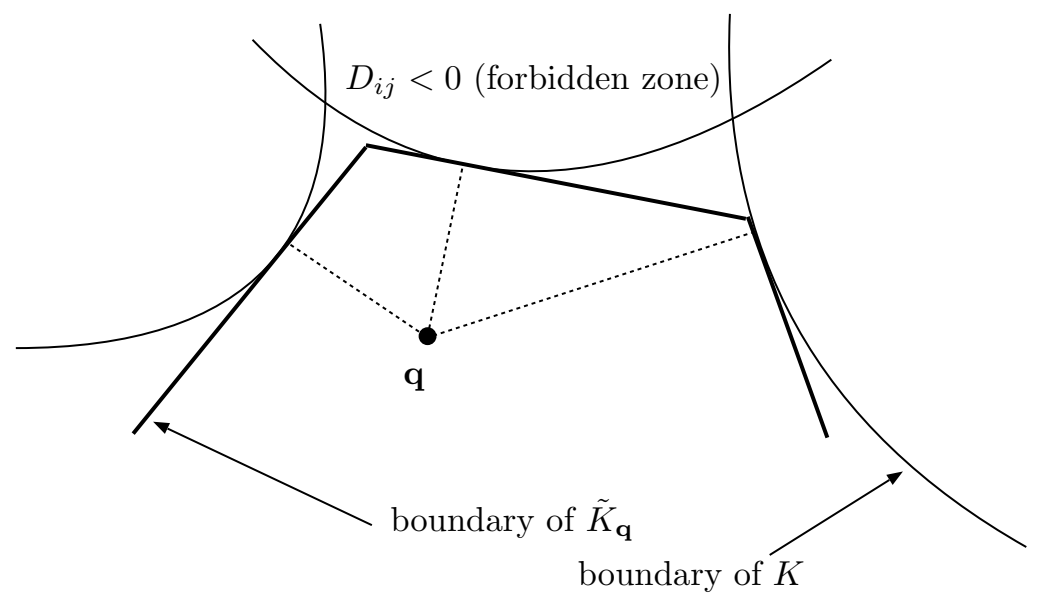

Figure 1. Inner approximation of the feasible set $K$.

$$
\tilde{K}_{\mathbf{q}}=\left\{\tilde{\mathbf{q}}, D_{i j}(\mathbf{q})+\mathbf{G}_{i j}(\mathbf{q}) \cdot(\tilde{\mathbf{q}}-\mathbf{q}) \geq 0 \quad \forall i<j\right\} .
$$

Thanks to the convex character of the function $D_{i j}$, it can be shown that $\tilde{K}_{\mathbf{q}} \subset K$ for any $\mathbf{q} \in K$, and it is a convex polyhedron as intersection of half spaces. The projection step (9) is replaced by

$$
\mathbf{q}^{n+1}=P_{\tilde{K}_{\mathbf{q}^{n}}}\left(\mathbf{q}^{n}+\tau \mathbf{U}\left(\mathbf{q}^{n}\right)\right),
$$

which amounts to project a predicted position $\tilde{\mathbf{q}}^{n+1}=\mathbf{q}^{n}+\tau \mathbf{U}\left(\mathbf{q}^{n}\right)$ on the

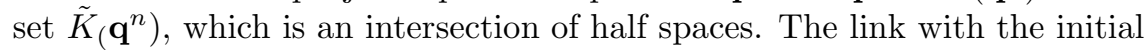
problem is more explicit if one expresses the scheme in terms of velocities. The approximate set of feasible velocities is defined by

$$
\tilde{C}^{\tau}(\mathbf{q})=\left\{\mathbf{v}, D_{i j}(\mathbf{q})+\tau \mathbf{G}_{i j}(\mathbf{q}) \cdot \mathbf{v} \geq 0 \quad \forall i<j\right\} .
$$

Now setting $\mathbf{u}^{n+1}=\left(\mathbf{q}^{n+1}-\mathbf{q}^{n}\right) / \tau$, the scheme (14) can be expressed in terms of velocities as

$$
\mathbf{u}^{n+1}=P_{\tilde{C}^{\tau}\left(\mathbf{q}^{n}\right)} \mathbf{U}\left(\mathbf{q}^{n}\right),
$$


which the discrete counterpart to (4). The problem can be put in a saddlepoint form:

$$
\begin{cases}\mathbf{u}+B^{\star} \lambda & =\mathbf{U} \\ B \mathbf{u} & \leq D / \tau \\ \lambda & \geq 0 \\ (B \mathbf{u}-D / \tau, \lambda) & =0 .\end{cases}
$$

where $D$ is the vector of distances at the current configuration, i.e. $\left(D_{i j}\left(\mathbf{q}^{n}\right)\right)$.

Remark 2.4. In the present context, the number of rows of $B$ is the number of potential contacts, i.e. $N(N-1) / 2$ ), whereas the number of rows of $B$ in the continuous setting (13) was the number of actual contacts, which is of the order of $3 N$. It is nevertheless possible to alleviate the computational costs by ruling out a priori the constraints that are not likely to become activated at the next step, which amounts to suppress the corresponding lines of $B$, thereby reducing the computational costs.

As it appears in Fig. 1, $K$ can hardly be seen as a global approximation of $\tilde{K}_{\mathbf{q}}$. Yet, if the time step is small, the constraints will be activated when $\mathbf{q}$ is closed to a forbidden zone, and in this case $\tilde{K}_{\mathbf{q}}$ approximates $K$ locally, i.e. in the neighborhood of $\mathbf{q}$. It can be proven that the scheme converges (Venel, 2011).

Uzawa algorithm Solutions to Problem (16) can be approximated by the Uzawa algorithm. Let $\lambda^{0}$ be given (the choice $\lambda=0$ can be made in case no prior information on $\lambda$ is available). Successive approximations $\left(\mathbf{u}^{k}, \lambda^{k}\right)$ are built as follows: once $\lambda^{k}$ is determined, $\mathbf{u}^{k+1}$ and $\lambda^{k+1}$ are defined by

$$
\begin{cases}\mathbf{u}^{k+1}+B^{\star} \lambda^{k} & =\mathbf{U} \\ \lambda^{k+1} & =\Pi_{+}\left(\lambda^{k}+\nu\left(B \mathbf{u}^{k+1}-D / \tau\right)\right)\end{cases}
$$

where $\Pi_{+}$is the euclidean projection on the cone of vectors with nonnegative components (a simple cut-off in practice), and $\nu>0$ is a fixed parameter.

Note that any fixed point of the algorithm is a solution to Problem (16). Indeed, consider $(\mathbf{u}, \lambda)$ such a fixed point,. For any component of $\lambda$ associated to a contact $(i, j)$, stationarity implies the following alternative:

i) either $\lambda_{i j}=0$ and $(B \mathbf{u}-D / \tau)_{i j} \leq 0$ (the constraint is satisfied, possibly in a strict sense, and the Lagrange multiplier is inactive), or

ii) $\lambda_{i j}>0$ and $(B \mathbf{u}-D / \tau)_{i j}=0$ (the constraint is saturated, and the Lagrange multiplier is active). 
The algorithm can be shown to converge as soon as $0<\nu<2 /\|B\|^{2}$ (see Ciarlet (1989)). This algorithm is actually a fixed-step algorithm of the projected gradient type, performed on the quadratic functional defined on the set of Lagrange multipliers:

$$
\mu \longmapsto \Psi(\mu)=\frac{1}{2}\left(\mathbf{U}-B^{\star} \mu, \mathbf{U}-B^{\star} \mu\right)+(\mu, D / \tau) .
$$

The quadratic part of the functional is the quadratic form associated to the matrix $B B^{\star}$, that is the discrete Laplace-like operator on the network already mentioned in the previous section. This very matrix will condition the numerical difficulty so numerically solve the system. Note that the present matrix $B B^{\star}$ actually differs from the one described in the previous section in that it pertains to all potential contacts, and not only actual ones (see Remark 2.4). Yet, in actual computations, active contacts for the discretization scheme will correspond to couples of particles that are not far away from each other, so that both matrices can be expected to share similar properties. The next section is dedicated to further remarks on this underlying Laplace-like operator, which reflects the microscopic arrangements of individuals.

\subsection{Underlying Laplace-like operator}

We investigate here the properties of the matrix $B B^{\star}$, which was identified as a kind of discrete Laplace operator. We shall see that this matrix actually differs from the matrices that result from space discretization of elliptic problems, or more generally from discrete Laplace operators for electric networks.

Let us consider a configuration $\mathbf{q} \in K$ (like in Fig. 2), and the associated matrix $B$, each line of which expresses the constraint

$$
-\mathbf{G}_{i j} \cdot \mathbf{u} \leq 0
$$

where $\mathbf{G}_{i j}$ is the gradient of the distance $D_{i j}=\left|\mathbf{q}_{j}-\mathbf{q}_{i}\right|-r_{i}-r_{j}$ with respect to the configuration vector $\mathbf{q}=\left(\mathbf{q}_{1}, \ldots, \mathbf{q}_{N}\right)$. Let us start with some comment on the operator $B^{\star}$. As we already pointed out, it can be interpreted as a discrete gradient. Considering a set of Lagrange multipliers $\lambda$ (i.e. interaction pressures between particles in contacts),$-B^{\star}$ assembles the corresponding force field acting on the particles. In case the configuration is structured (e.g. orthogonal lattice of discs of the same size, or the triangular lattice of Fig.. 3) then a constant pressure field induces no force at all (expect for the boundary of the cluster). It is the discrete counterpart of the standard property in the continuous setting that the gradient of 


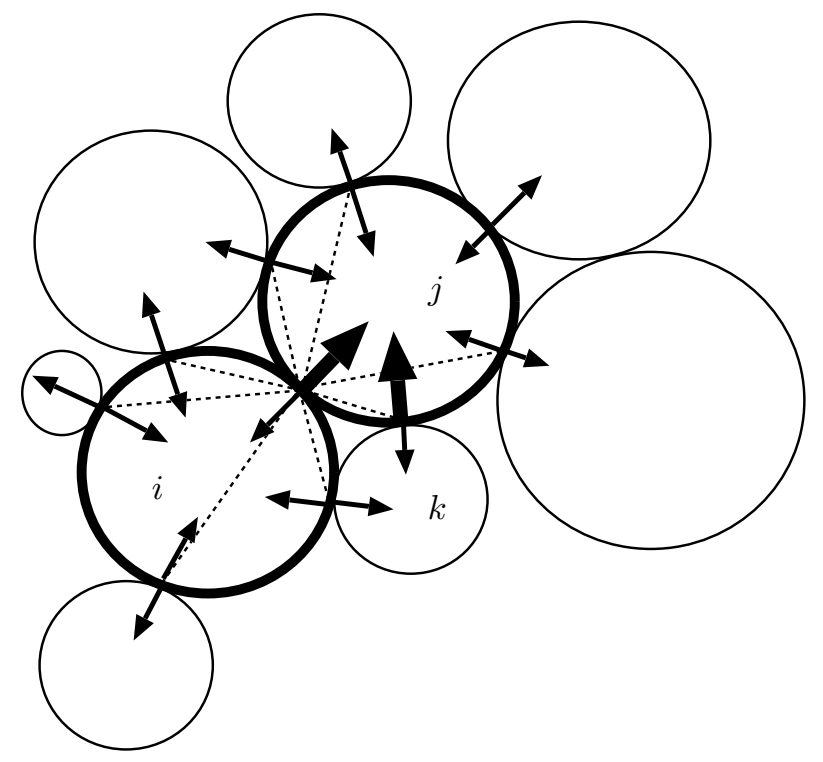

Figure 2. Non structured stencil

a constant field is zero. Yet, in the general situation (when no structural assumption is made on the local arrangements of discs), this property does not hold. See e.g. the discs on Fig. 2: the vectors pointing inward each particle do not sum up to zero. Another feature is typical of the unstructured discrete situation. Consider the cluster represented in Fig. 3. The number of discs is 14 , thus the number of degrees of freedom is 28 , whereas the number of contacts is 29 . As a consequence, the kernel of $B^{\star}$ is not trivial: there exists a non-zero pressure field (one pressure for each contact point) such that the resulting force field is 0 . A striking consequence of this fact is the following: the associated Laplace-like operator $B B^{\star}$ does not satisfy the Hopf maximum principle, since there exist pressure fields $\lambda$ such that $B B^{\star} \geq 0$, whereas some pressures are negative.

The matrix $B B^{\star}$ can be expressed as follows: considering a pressure field $\lambda=\left(\lambda_{k \ell}\right)$, where $(k, \ell)$ runs over the set of actual contacts, the vector $B B^{\star} \lambda$ is a pressure-like vector (one component for each contact), and the value corresponding to the contact between $\operatorname{discs} i$ and $j$ is

$$
\sum_{(k, \ell) \sim(i, j)} \lambda_{k \ell} \mathbf{G}_{i j} \cdot \mathbf{G}_{k \ell} .
$$




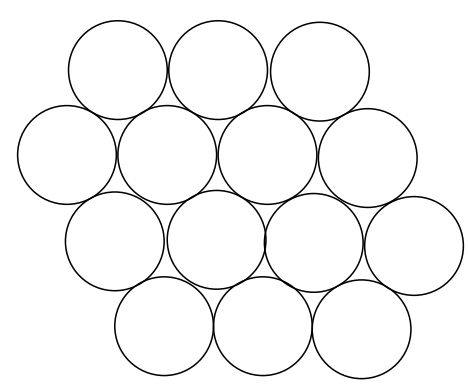

Figure 3. Hyperstatic situation

The discrete matrix operates on the network which is dual to the primary network (i.e. the network made of the centers of discs, with a connection between two vertices as soon as the discs are in contact). The vertices of the dual network are the contact points, and two vertices are connected, or equivalently the corresponding element of the matrix is non zero, as soon as the contacts share a same particle. Both networks are represented in Fig. 4. The corresponding stencil is represented in Fig. 2, around a vertex that is the contact point between two particles. The non-verification of the maximum principle is due to the fact that, when one considers three particles $i, j$, and $k$, it may happen that

$$
\mathbf{e}_{i j} \cdot \mathbf{e}_{k j}>0
$$

where $\mathbf{e}_{i j}$ is the unit vector $\left(\mathbf{q}_{j}-\mathbf{q}_{i}\right) /\left|\mathbf{q}_{j}-\mathbf{q}_{i}\right|$. Examples of such vectors are represented in Fig. 2, in bold. This property is generic in jammed collections of discs. As a consequence, some of the extra diagonal elements of $B B^{\star}$ are positive, thus $B B^{\star}$ is not a $M$-matrix. If one aims at interpreting the situation in terms of electric networks, in means that some resistances are negative, and this situation is native for jammed population of individual identified to rigid discs.

Condition number In terms of numerical computation, the inner difficulty of the problem can be quantified by the condition number of $B B^{\star}$ (i.e. the ratio between the largest and the smallest non zero eigenvalue of this symmetric positive matrix). This number $\kappa$ can be related in some way to the lack of convexity of the feasible set $K$ in the following sense: as detailed 

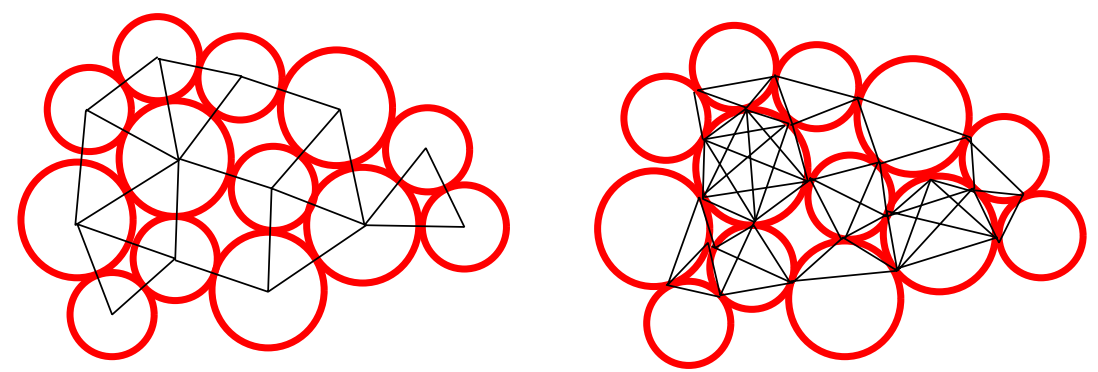

Figure 4. Primal (left) and dual (right) contact networks

in Maury and Venel (2011), it holds that

$$
\kappa=\operatorname{cond}_{2}\left(B B^{\star}\right) \geq \frac{C}{\eta^{2}},
$$

where $\eta$ is the prox-regularity constant of $K$, i.e. the largest constant that can be chosen in Definition 2.3. This constant tends to zero very fast as the number of discs grows (see again Maury and Venel (2011)).

\section{Macroscopic setting}

\subsection{The macroscopic model}

We aim now at describing the population at a macroscopic level, i.e. by a density $\rho(x, t)$. We consider a bidimensional domain $\Omega$ (the room), we denote by $\mathbf{U}$ the desired velocity field (which is given), and by $\mathbf{u}$ the actual velocity field (which is affected by congestion effects). The set of feasible densities is defined as

$$
K=\left\{\rho \in L^{1}(\Omega), 0 \leq \rho(x) \leq 1 \quad \text { a.e. in } \Omega\right\} .
$$

The density is transported by the actual velocity field ${ }^{4}$ :

$$
\frac{\partial \rho}{\partial t}+\nabla \cdot(\rho \mathbf{u})=0
$$

where $\mathbf{u}$ is defined as the $L^{2}$ projection of $\mathbf{U}$ on the cone of feasible velocities $C_{K}(\rho)$. This model was proposed in Maury et al. (2010), and studied in the 
case where $\mathbf{U}$ is a gradient.

Unformally said, $C_{K}(\rho)$ contains velocity fields that do not increase the density where the constraint is already saturated (i.e. wherever $\rho=1$ ). It can be defined in a dual way as

$$
\begin{gathered}
C_{K}(\rho)=\left\{\mathbf{v} \in L^{2}(\Omega)^{2}, \int_{\Omega} \mathbf{v} \cdot \nabla q \leq 0 \quad \forall q \in H_{+}^{1}(\Omega), \int_{\Omega} q(1-\rho)=0\right\}, \\
\text { with } H_{+}^{1}(\Omega)=\left\{q \in H^{1}(\Omega), q \geq 0 \text { a.e. in } \Omega\right\} .
\end{gathered}
$$

Note that the constraint on the integral of $q(1-\rho)$ imposes that $q$ is 0 in the zones where the constraint is not saturated (i.e. $\rho<1$ ).

When a part $\Sigma$ of the boundary of $\Omega$ is an open exit, a free outlet condition can be prescribed by simply prescribing that the pressure is 0 on $\Sigma$ (since the pressure is in $H^{1}(\Omega)$, its trace on $\Sigma$ is indeed well-defined).

Saddle-point formulation The projection of the desired velocity on the cone of feasible ones can be formulated in a dual manner, in the form of a unilateral Darcy problem:

Find $(\mathbf{u}, p) \in L^{2}(\Omega)^{2} \times H_{\rho}^{1}(\Omega)$, where

$$
H_{\rho+}^{1}(\Omega)=\left\{q \in H_{+}^{1}(\Omega), \int_{\Omega} q(1-\rho)=0\right\}
$$

such that

$$
\left\{\begin{array}{l}
\mathbf{u}+\nabla p=\mathbf{U} \\
-\int_{\Omega} \mathbf{u} \cdot \nabla q \leq 0
\end{array} \text { for all } q \in H_{\rho+}^{1}(\Omega),\right.
$$

with the complementarity condition:

$$
-\int_{\Omega} \mathbf{u} \cdot \nabla p=0 .
$$

Toward a suitable framework The model can be written

$$
\frac{\partial \rho}{\partial t}+\nabla \cdot(\rho \mathbf{u}(\rho))=0
$$

\footnotetext{
${ }^{4}$ The transport equation is meant in a weak sense; we shall say that $\mathbf{u}$ transports $\rho$ in the time interval $[0, T]$, with initial condition $\rho=\rho^{0}$, when$$
\int_{0}^{T} \int_{\Omega} \rho \partial_{t} \varphi+\int_{0}^{T} \int_{\Omega} \rho \mathbf{u} \cdot \nabla \varphi+\int_{\Omega} \varphi(0, \cdot) \rho^{0}=0 \quad \forall \varphi \in C_{c}^{\infty}([0, T) \times \Omega) .
$$ 
where the mapping $\rho \mapsto \mathbf{u}(\rho)$ corresponds to the projection of $\mathbf{U}$ on $C_{K}(\rho)$. This mapping is nonlinear, nonlocal, the dependence upon $\rho$ is not smooth; it does not fit in the classical framework of conservation laws.

In the microscopic setting, the difference $\tilde{\mathbf{q}}-\mathbf{q}$ between the configurations $\mathbf{q}$ and $\tilde{\mathbf{q}}$ corresponds to a collection of individual displacements which reflects the Lagrangian character of the description. In the standard framework of Partial Differential Equations, the difference between entities (or, more generally, functions) $\tilde{\rho}-\rho$ is of different nature, because of the Eulerian character of the description. In order to extend the tools we used in the microscopic context, we must adopt another standpoint. Let us show that the Wasserstein distance, based on optimal transportation, provides an adapted framework.

\subsection{Optimal transportation and Wasserstein distance}

We present here some basics on optimal transportation, and we refer to Villani (1995) for a more detailed and more general presentation of those concepts. We assume here that the domain $\Omega$ is convex. For any measurable map $\mathbf{t}: \Omega \longrightarrow \Omega$, and probability densities ${ }^{5} \mu$ and $\nu$ supported in $\bar{\Omega}$, we say that $\nu$ is the pushforward of $\mu$ by $\mathbf{t}$ whenever

$$
\int_{\mathbf{t}^{-1}(A)} \mu(x) d x=\int_{A} \nu(x) d x
$$

for any measurable set $A \subset \Omega$. Considering that the cost of moving $x$ to $y$ is $|y-x|^{2}$ (quadratic cost), the cost of the transport map $\mathbf{t}$ is defined as

$$
C(\mathbf{t})=\int_{\Omega}|\mathbf{t}(x)-x|^{2} d x .
$$

The quadratic Wasserstein distance $W_{2}(\mu, \nu)$ is then defined by

$$
W_{2}(\mu, \nu)^{2}=\inf _{\mathbf{t}, \mathbf{t}_{\sharp} \mu=\nu} C(\mathbf{t})=\inf _{\mathbf{t}, \mathbf{t}_{\sharp} \mu=\nu} \int_{\Omega}|\mathbf{t}(x)-x|^{2} d x .
$$

In the case we considered (in particular the first measure is absolutely continuous), the minimizer is attained, and the minimization problem can be formalized in a dual way: it holds that

$$
\frac{1}{2} W_{2}(\mu, \nu)^{2}=\frac{1}{2} \min _{\mathbf{t}, \mathbf{t}_{\sharp} \mu=\nu} \int_{\Omega}|\mathbf{t}(x)-x|^{2} d x
$$

\footnotetext{
${ }^{5}$ As detailed in Villani (1995), the approach generalizes to general measures, but we assume here absolute continuity, which is the case in the situation we consider.
} 
$=\max _{\varphi, \psi \in C_{b}(\Omega)}\left\{\int_{\Omega} \varphi(x) \rho(x) d x+\int_{\Omega} \psi(y) \nu(y) d y, \varphi(x)+\psi(y) \leq \frac{1}{2}|y-x|^{2}\right\}$,

where $C_{b}(\Omega)$ is the space of all those functions that are bounded and continuous in $\Omega$. The latter maximum is attained for a couple $\left(\varphi, \varphi^{c}\right)$, where

$$
\varphi^{c}(y)=\inf _{x}\left(\frac{1}{2}|x-y|^{2}-\varphi(x)\right) .
$$

The function $\varphi$ is called a Kantorovich potential for the transport problem from $\mu$ to $\nu$; it is related to the transport map $\mathbf{t}$ that realizes the Wasserstein distance by

$$
\mathbf{t}=\boldsymbol{i}-\nabla \varphi
$$

where $\boldsymbol{i}$ is the identity. Let us consider a toy problem to illustrate the considerations above. Let $\eta$ be given in $(0,1 / 2)$, and let $I_{\eta}$ be the interval $(-1 / 2-\eta / 2,1 / 2+\eta / 2) \subset \mathbb{R}$, the length of which is $1-\eta$. We consider a probability density

$$
\rho_{\eta}=\frac{1}{1-\eta} \mathbf{1}_{I_{\eta}},
$$

(characteristic function of $I_{\eta}$, normalized to recover a unit mass). The density $\rho_{\eta}$ violates the constraint and its projection on $K$ is $\rho_{0}$, the characteristic function of the interval $(-1 / 2,1 / 2)$. The Kantorovich potentials (from $\rho_{0}$ to $\rho_{\eta}$ ) can be computed exactly as

$$
\varphi(x)=\frac{\eta}{2} x^{2}, \Psi(y)=-\frac{1}{2} \frac{1}{1-\eta} y^{2} .
$$

The transport map from $\rho_{0}$ to $\rho_{\eta}$ is indeed

$$
x \longmapsto(i-\nabla \varphi) x=(1-\eta) x,
$$

and its inverse $\left(\rho_{\eta}\right.$ to $\left.\rho_{0}\right)$ is

$$
y \longmapsto(i-\nabla \psi) y=y+\frac{\eta}{1-\eta} y=\frac{1}{1-\eta} y .
$$

\subsection{Catching-up algorithm}

Let $\tau>0$ be a time step. The catching-up algorithm consists in transporting the current density by the desired velocity field, and then projecting it on the set $K$ of feasible densities:

$$
\tilde{\rho}^{n+1}=(\boldsymbol{i}+\tau \mathbf{U})_{\#} \rho^{n} \quad(\text { prediction step}),
$$




$$
\rho^{n+1}=P_{K} \tilde{\rho}^{n+1} \quad(\text { correction step }),
$$

where the projection is performed in the Wasserstein sense. This projection can be shown to be well defined ${ }^{6}$ (Maury et al., 2011).

In the microscopic setting, the link between the catching-up algorithm and the crowd motion model was straightforward ${ }^{7}$. In the present situation, the link is less straightforward, and the proof of convergence of the discrete trajectories requires technical developments that we will not describe here (see Maury et al. (2010, 2011)). We shall simply describe here the core of the proof, which lies in the link between the catching up scheme and the unilateral Darcy problem (20).

In the microscopic setting, the actual velocity at the discrete level was written $\left(\mathbf{q}^{n+1}-\mathbf{q}^{n}\right) / \tau$ (see e.g. (8)). This expression has to replaced by a similar expression involving $\rho^{n}$ and

$$
\rho^{n+1}=P_{K}\left((\boldsymbol{i}+\tau \mathbf{U})_{\#} \rho^{n}\right),
$$

which would be a Lagrangian version of " $\left(\rho^{n+1}-\rho^{n}\right) / \tau$ ", so to say. The

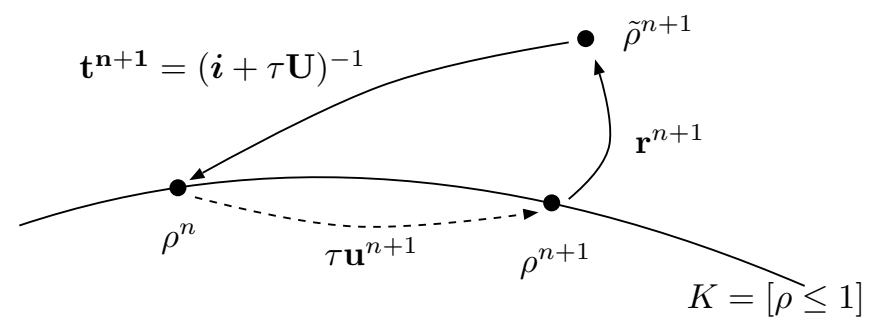

Figure 5. Definition of the discrete transport maps.

situation is represented in Fig. 5. The main idea consists in defining a

\footnotetext{
${ }^{6}$ This fact is somewhat striking since, in the microscopic setting, the set $K$ can be shown to be $\eta$-prox-regular with $\eta$ going to 0 as the number of individuals goes to $\infty$, and their size goes to 0 . It means that the projection is defined in a neighborhood of $\partial K$ which shrinks down to $\partial K$ itself as the population grows, which suggests a degenerated behavior when it tends to the macroscopic situation. The very fact that it does not degenerate in the macroscopic situation (the projection is well-defined, no matter what the distance to $K$ is) reflects the deep difference between the two approaches, as it was already addressed at the end of Section 1 .

${ }^{7}$ We refer the reader to the few lines between Eq. (8) (which is a discretization of (5)) and Eq. (9)
} 
discrete velocity $\mathbf{u}^{n+1}$ such that $\tau \mathbf{u}^{n+1}$ corresponds to the displacement between $\rho^{n}$ and $\rho^{n+1}$, and to show that this velocity solves (at least at the first order in time) a Darcy problem like (20). It can be done as follows: we define $\mathbf{t}^{\mathbf{n}+\mathbf{1}}$ as $(\boldsymbol{i}+\tau \mathbf{U})^{-1}$, which is well defined as soon as $\mathbf{U}$ is regular and $\tau$ is small enough, and $\mathbf{r}^{n+1}$ as the optimal map between $\rho^{n+1}$ and $\tilde{\rho}^{n+1}$. The discrete velocity is then defined as

$$
\mathbf{u}^{n+1}=\frac{\boldsymbol{i}-\mathbf{t}^{\mathbf{n}+\mathbf{1}} \circ \mathbf{r}^{n+1}}{\tau}
$$

Notice that this velocity is defined in the target set: considering a element of mass $x$ of $\rho^{n}$, transported to $y$ in $\rho^{n+1}$, it holds

$$
y=x+\tau \mathbf{u}^{n+1}(y),
$$

where the velocity is defined at $y$, and not at $x$.

Let us introduce

$$
\mathbf{w}^{n+1}=\frac{i-\mathbf{r}^{n+1}}{\tau} \Longleftrightarrow \mathbf{r}^{n+1}=\boldsymbol{i}-\tau \mathbf{w}^{n+1} .
$$

As soon as $\mathbf{U}$ is assumed to be smooth (i.e. continuously differentiable), $\mathbf{t}^{n+1}$ can be expanded at the first order as

$$
\mathbf{t}^{n+1}=(\boldsymbol{i}+\tau \mathbf{U})^{-1}=\boldsymbol{i}-\tau \mathbf{U}+o(\tau),
$$

where the $o(\tau)$ if uniform with respect to the space variable $x$. We then have

$$
\begin{aligned}
\mathbf{u}^{n+1}=\frac{1}{\tau}(\boldsymbol{i} & \left.-(\boldsymbol{i}-\tau \mathbf{U}+o(\tau)) \circ\left(\boldsymbol{i}-\tau \mathbf{w}^{n+1}\right)\right) \\
= & \mathbf{w}^{n+1}+\mathbf{U}+O(\tau)
\end{aligned}
$$

To complete the identification with (20), we still have to establish that $\mathbf{w}^{n+1}$ is the gradient of a pressure $p$ that is nonnegative, and that vanishes outside the saturated zone. To that purpose, we follow the strategy introduced in Butazzo and Santambrogio (2005) and used in the context of crowd motion models of the gradient flow type in Maury et al. (2010). It consists in proving that the Kantorovich potential associated to the transport problem from $\rho^{n+1}$ to $\tilde{\rho}^{n+1}$ (which corresponds to the displacement $-\tau \mathbf{w}^{n+1}$ ) can be interpreted (up to multiplicative and additive constants) as a pressure field in the saturated zone. We shall assume here that $\tilde{\rho}^{n+1}$ is positive in $\Omega$ (we refer to Maury et al. (2010) for an adaptation to the general case). The proof is somewhat paradoxical, since it consists in considering eulerian variations around the minimizer, whereas the Wasserstein framework suggests 
to build variations by means of transport maps (i.e. horizontal variations). Consider $\mu$ a density in $K$, and $\varepsilon>0$. We define

$$
\rho_{\varepsilon}=\rho^{n+1}+\varepsilon\left(\mu-\rho^{n+1}\right) \in K,
$$

and we denote by $\varphi_{\varepsilon}$ and $\psi_{\varepsilon}$ the Kantorovich potentials associated to the transport problem from $\rho_{\varepsilon}$ to $\tilde{\rho}^{n+1}$. By the Monge Kantorovich formulation (21), it holds that

$$
\frac{1}{2} W_{2}\left(\rho_{\varepsilon}, \tilde{\rho}^{n+1}\right)^{2}=\int_{\Omega} \varphi_{\varepsilon} \rho_{\varepsilon}+\int_{\Omega} \psi_{\varepsilon} \tilde{\rho}^{n+1},
$$

and

$$
\begin{gathered}
\frac{1}{2} W_{2}\left(\rho^{n+1}, \tilde{\rho}^{n+1}\right)^{2}= \\
\max _{\varphi, \psi \in C_{b}(\Omega)}\left\{\int_{\Omega} \varphi \rho^{n+1}+\int_{\Omega} \psi \tilde{\rho}^{n+1}, \varphi(x)+\psi(y) \leq \frac{1}{2}|y-x|^{2}\right\} \\
\geq \int_{\Omega} \varphi_{\varepsilon} \rho^{n+1}+\int_{\Omega} \psi_{\varepsilon} \tilde{\rho}^{n+1} .
\end{gathered}
$$

Besides, since $\rho^{n+1}$ minimizes the Wassertein distance from $\tilde{\rho}^{n+1}$ to $K$ by construction, and since $\rho_{\varepsilon}$ is in $K$, we have

$$
\frac{1}{2} W_{2}\left(\rho_{\varepsilon}, \tilde{\rho}^{n+1}\right)^{2} \geq \frac{1}{2} W_{2}\left(\rho^{n+1}, \tilde{\rho}^{n+1}\right)^{2}
$$

which yields, thanks to (26) and (27),

$$
\int_{\Omega} \varphi_{\varepsilon}\left(\rho_{\varepsilon}-\rho^{n+1}\right) \geq 0 \Longrightarrow \int_{\Omega} \varphi_{\varepsilon} \rho^{n+1} \leq \int_{\Omega} \varphi_{\varepsilon} \mu \quad \forall \mu \in K .
$$

Now, prescribing a fixed value of the Kantorovich potentials at some point $x_{0} \in \Omega$, the Kantorovich potential is unique, and the sequence $\left(\varphi_{\varepsilon}\right)$ can be shown (see Butazzo and Santambrogio (2005), Lemma 3.4) to converge to the Kantorovich potential $\varphi$ associated to the transport from $\rho^{n+1}$ to $\tilde{\rho}^{n+1}$ (i.e. in the limit $\varepsilon=0$ ). So finally $\rho^{n+1}$ minimizes a linear functional of the type

$$
\rho \longmapsto \int_{\Omega} \varphi \rho
$$

over $K$ which is the set of probability densities that are less that 1 almost everywhere. If the upper bound constraint were not prescribed, $\rho$ would tend to concentrate on the minimizer(s) of $\varphi$. This concentration is ruled 


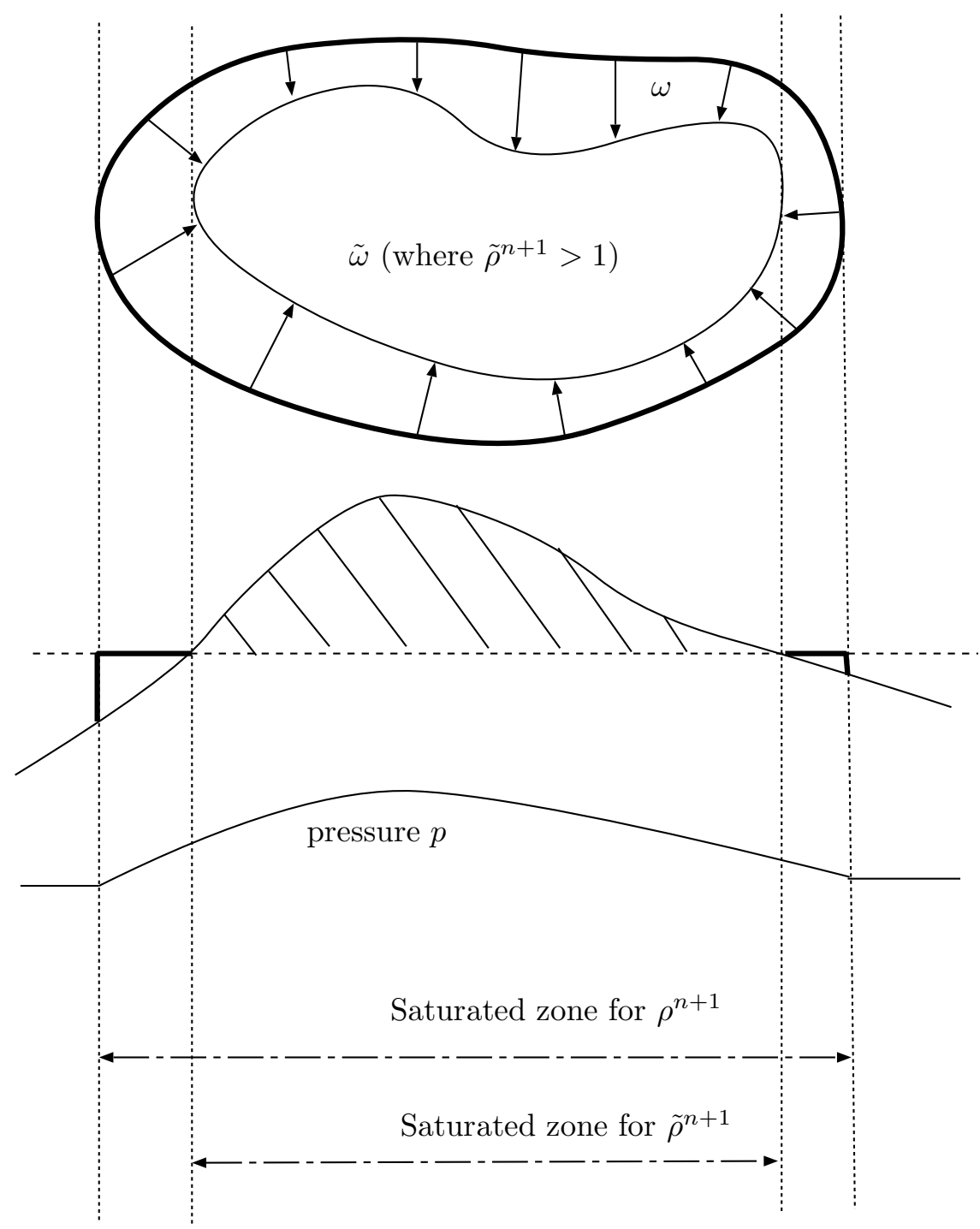

Figure 6. Projection of $\tilde{\rho}$ onto $K$ 
out by the congestion constraint, and $\rho$ saturates the zone in which $\varphi$ is minimal, so that it takes the following form

$$
\rho^{n+1} \mid \begin{array}{lll}
=1 & \text { on } & {[\varphi<\ell]} \\
\leq 1 & \text { on } & {[\varphi=\ell]} \\
=0 & \text { on } & {[\varphi>\ell]}
\end{array}
$$

where $\ell$ is a value which is adjusted to comply with the unit mass constraint. In the previous expression, the way $\rho^{n+1}$ is distributed over $[\varphi=\ell]$ is underdetermined, since it does not affect the value of the functional: the solution of the previous minimization problem is not unique. It does not matter here, since $\rho^{n+1}$ was already identified as the unique density that realizes the distance to $K$. We then define the pressure field

$$
p=\frac{1}{\tau}(\ell-\varphi)^{+} .
$$

By construction, $p$ is nonnegative, it vanishes outside of the saturated zone (i.e. where $\rho^{n+1}<1$ ), and $\nabla p=-\nabla \varphi / \tau$ in the support of $p$. Now recall that $\varphi$ is a Kantorovich potential for $\mathbf{r}^{n+1}$, i.e.

$$
\mathbf{r}^{n+1}=\boldsymbol{i}-\nabla \varphi=\boldsymbol{i}+\tau \nabla p .
$$

Finally, we have

$$
\mathbf{w}^{n+1}=\frac{\boldsymbol{i}-\mathbf{r}^{n+1}}{\tau}=-\nabla p,
$$

so that, thanks to (25), we obtain the Darcy decomposition (at the first order in time) of the desired velocity field $\mathbf{U}$ as the sum of the actual velocity and the gradient of a pressure:

$$
\mathbf{u}^{n+1}+\nabla p=\mathbf{U}+O(\tau) .
$$

Fig. 6 is an attempt to illustrate this construction: the zone in which the constraint is violated (i.e. $\tilde{\rho}^{n+1}>1$ ) is denoted by $\tilde{\omega}$. The excess of mass is spread out around this zone, and the obtained density saturates the constraint $\left(\rho^{n+1} \equiv 1\right)$ on $\omega$. Note again that the displacement $\tau \mathbf{u}^{n+1}$ which pushes $\rho^{n}$ onto $\rho^{n+1}$ is defined on the target set, which rules out a straight use of this approach to design a tractable numerical procedure.

\subsection{Numerical issues}

Like in the microscopic setting, a direct use of the saddle point form of the projection onto $K$ is delicate, since the pressure field is defined on the target set. We describe here a Monte Carlo algorithm based on a stochastic 
interpretation of the Poisson problem with Dirichlet boundary condition (see Maury et al. (2011) for details). Let us make it clear that this approach is not covered by a rigorous numerical analysis. We consider again the situation represented in Fig. 5, and we drop the upperscripts ${ }^{n+1}$ to alleviate notation. The predicted density $\tilde{\rho}$ violates the constraint in the domain $\tilde{\omega}$, and the excess mass is $(\tilde{\rho}-1)^{+}$. Since this excess mass is due to the transport of an admissible density by a contracting field (desired velocity field $\mathbf{U}$ ) during $\tau$, it is of the order $\tau$. Let us assume that it can be written $\tau \nu$, where $\nu$ is a nonnegative density supported by the oversaturated zone ${ }^{8}$.

The density $\tilde{\rho}$ is $1+\tau \nu$ in $\tilde{\omega}$. Let $\rho$ be the projection of $\tilde{\rho}$ on $K$. The displacement from $\rho$ to $\tilde{\rho}$ is of the form $\mathbf{r}=\boldsymbol{i}+\tau \nabla p$, where $p$ is 0 outside $\omega$ (see the previous section). Since $\rho$ saturates the constraint ${ }^{9}$ in $\omega$, we have in $\omega$

$$
\frac{1}{|\boldsymbol{i}+\tau \nabla p|}(x) \approx(1-\tau \nabla \cdot \nabla p)(x)=1+\tau \nu(\mathbf{r}(x)),
$$

so that, at the first order in $\tau$,

$$
-\Delta p(x)=\nu(\mathbf{r}(x))
$$

with $p=0$ on the boundary of $\omega$. The unknown pressure field $p$ and the right-hand side are not defined on the same set, but $\mathbf{r}$ is close to the identity $\boldsymbol{i}$. Replacing $\nu(\mathbf{r}(x))$ by $\nu(x)$ is audacious, even in this informal approach, since $\nu$ has no reason to be regular. Yet, $\nu$ and $\nu \circ \mathbf{r}$ are close (of the order $\tau$ ) in the Wasserstein sense by construction, therefore their distance in the $H^{-1}$ sense is of the same order (see Maury et al. (2010), Lemma 3.4). Thus, the corresponding pressures are also close (at the first order in $\tau$ ) in the $H^{1}$ norm. Using again the fact that $\omega$ is transported to $\tilde{\omega}$ by $\mathbf{r}$, which is the identity at the first order in $\tau$, and assuming sufficient regularity to transport the elliptic problem back to $\tilde{\omega}$ (up to first order terms), we end up with a Poisson problem in $\tilde{\omega}$

$$
-\Delta p=\nu \quad \text { in } \tilde{\omega},
$$

with homogeneous Dirichlet boundary conditions on $\partial \tilde{\omega}$.

The velocity of the boundary is $-\partial p / \partial n$, which means that the quantity of mass crossing an element $d \gamma$ of the boundary during $\tau$ is $-\tau \partial p / \partial n d \gamma$. Now consider the stochastic interpretation of the Poisson problem (we assume that $\nu$ has unit mass, which can be recovered by a straightforward

\footnotetext{
${ }^{8}$ Since $\tilde{\rho}$ comes from the transport of the previous density $\rho^{n}$ by $\mathbf{U}$, it holds approximately $\tilde{\rho} \approx 1-\tau \nabla \cdot \mathbf{U}+o(\tau)$.

${ }^{9}$ This property is straightforward: if it were not, it would obviously not be the density closest to $\tilde{\rho}$ in $K$.
} 
renormalization): consider a brownian motion starting at an initial point $X$, which is supposed to be itself a random position following the law of density $\nu$ in $\tilde{\omega}$, and consider the location at which this Brownian motion crosses the boundary of $\tilde{\omega}$. The law of the position of this first hit is known to follow a law with density $-\partial p / \partial n$ on $\partial \tilde{\omega}$, where $p$ is the solution to the Poisson problem above. The algorithm we propose (see Maury et al. (2011)) is deduced from these consideration.

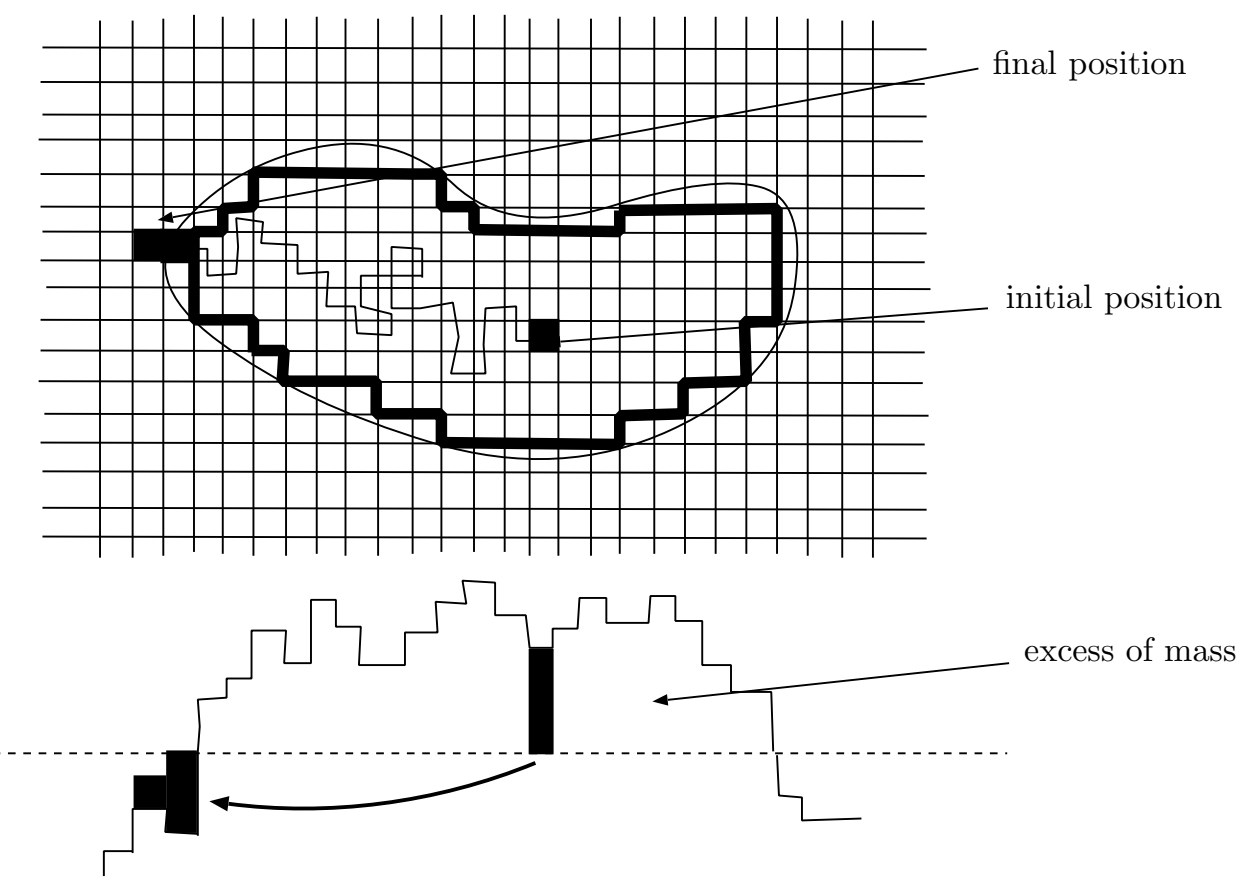

Figure 7. Random walk algorithm

The space is discretized in a finite volume spirit by a cartesian mesh. The densities are assumed to be constant on each cell of this mesh. We illustrate in Fig. 7 the discretized counterpart of the situation represented in Fig. 6 . The zone delimited by the bold broken line corresponds to cells where the constraint is violated. Considering one of those cells as a starting point, with an excess of mass $m$, we run a random walk on the grid with balanced transition probabilities. When the random walk reaches a cell which is not saturated, the excess of mass is put in this cell, up to saturation. If there is not enough empty space to get rid of the total excess $m$, the random 
walk continues according to the same principle, until there is no mass left. Fig. 7 illustrates the process, starting from a cell inside the zone where the constraint is violated.

A new random walk is then initiated from another cell where the constraint is saturated, and so on, until the constraint is satisfied everywhere. We refer to Maury et al. (2011) for a more detailed description of the algorithm, and for some illustrations of the behavior of the algorithm.

\section{Bibliography}

H. H. Bauschke and P. L. Combettes. Convex analysis and monotone operator theory in Hilbert spaces. CMS Books in Mathematics/Ouvrages de Mathématiques de la SMC. Springer, New York, 2011.

G. Butazzo and F. Santambrogio. A model for the optimal planning of an urban area. SIAM J. Math. Anal., pages 514-530, 2005.

P. G. Ciarlet. Introduction to Numerical Linear Algebra and Optimisation. Cambridge Texts in Applied Mathematics. Cambridge University Press, Cambridge, 1989.

F. H. Clarke, R. J. Stern, and P. R. Wolenski. Proximal smoothness and the lower-C2 property. J. Convex Anal., pages 117-144, 1995.

B. Maury. A time-stepping scheme for inelastic collisions. numerical handling of the nonoverlapping constraint. Numerische Mathematik, 102: 649-679, 2006.

B. Maury and J. Venel. A discrete contact model for crowd motion. ESAIM Mathematical Modelling and Numerical Analysis, 45:145-168, 2011.

B. Maury, A. Roudneff-Chupin, and F. Santambrogio. A macroscopic crowd motion model of gradient flow type. Mathematical Models and Methods in Applied Sciences, 20:1787-1821, 2010.

B. Maury, A. Roudneff-Chupin, F. Santambrogio, and J. Venel. A macroscopic crowd motion model of gradient flow type. Networks and Heterogeneous Media, 6(3):485-519, September 2011.

J.-J. Moreau. Décomposition orthogonale d'un espace Hilbertien selon deux cônes mutuellement polaires. C. R. Acad. Sci. Paris, 255:238-240, 1962.

J.-J. Moreau. Evolution problem associated with a moving convex set in a hilbert space. J. Differential Equations, 26:346-374, 1977.

R. A. Poliquin and R. T. Rockafellar. Prox-regular functions in variational analysis. Trans. Amer. Math. Soc., 348:1805-1838, 1996.

R.T. Rockafellar. Convex Analysis. Princeton mathematical series. Princeton University Press, 1970.

J. Venel. A numerical scheme for a class of sweeping processes. Numerische Mathematik, 118:367-400, 2011.

C. Villani. Topics in Optimal Transportation, volume 58. AMS, 1995. 